\title{
Preventing and exploiting the oncogenic potential of integrating gene vectors
}

\author{
Ute Modlich ${ }^{1}$ and Christopher Baum ${ }^{1,2}$
}

'Department of Experimental Hematology, Hannover Medical School, Hannover, Germany. 2Division of Experimental Hematology, Cincinnati Children's Hospital Medical Center, Cincinnati, Ohio, USA.

\begin{abstract}
Gene therapy requires efficient gene delivery to cure or prevent disease by modifying the genome of somatic cells. However, gene vectors, which insert themselves into the host genome in order to achieve persistent protein expression, can trigger oncogenesis by upregulating cellular protooncogenes. This adverse event, known as insertional mutagenesis, has become a major hurdle in the field. Vectors developed on the basis of lentiviruses are considered to be less genotoxic than the hitherto used $\gamma$-retroviral vectors. For their report in this issue of the JCI, Montini et al. utilized a tumor-prone mouse model to identify the genetic determinants of insertional mutagenesis (see the related article beginning on page 964). They report that the lentiviral integration pattern and additional improvements in vector design reduce the genotoxic risk. These findings will inform future vector design with the goal of limiting genotoxicity for gene therapy or increasing genotoxicity for protooncogene discovery.
\end{abstract}

The development of safe and efficient methods to modify the genome of somatic cells would provide new perspectives in the prevention, treatment, or even causal cure of inherited and acquired diseases. Gene vectors that insert themselves into the host genome would allow for a longlasting phenotypic correction even if the target cells undergo repeated rounds of cell division. Retroviruses and their derived vectors show a highly efficient yet semirandom integration pattern in the host genome. The simple $\gamma$-retroviruses have a propensity to integrate into transcriptional start sites and regulatory gene regions (1), with a surprising preference for genes involved in proliferative signal cascades (2). Insertional mutagenesis by replication-competent murine leukemia virus, a prototypic $\gamma$-retrovirus, thus became a powerful tool for protooncogene discovery (3). Still, for replicationdeficient $\gamma$-retroviral gene vectors, it was not until 18 years after the first successful modification of hematopoietic stem cells in 1984 that the leukemogenic potential of insertional mutagenesis was demon-

Conflict of interest: The authors have declared that no conflict of interest exists.

Nonstandard abbreviations used: $C d k n 2 a$, cyclindependent kinase inhibitor 2A; HIV-1, HIV type 1; LTR, long terminal repeat; SIN, self-inactivating.

Citation for this article: J. Clin. Invest. 119:755-758 (2009). doi:10.1172/JCI38831. strated $(4,5)$. Subsequently, clinical gene therapy studies have underlined the danger of this complication, which manifests in a highly context-dependent manner and likely involves the acquisition of secondary mutations and variable selection pressure of the underlying disease (6-12).

Compared with $\gamma$-retroviruses, lentiviruses such as HIV type 1 (HIV-1) and their derived vectors show a stronger preference for integrating within active transcription units without an obvious bias for proliferation-associated genes or transcriptional start sites, which suggests a lower potential for triggering oncogenic adverse events by insertional mutagenesis $(1,13)$. The rationale for the therapeutic use of HIV-1-based vectors originated from their evolutionary background as a highly efficient human-infectious, nononcogenic retrovirus that may even transduce nondividing cells (14). Lentiviral vectors have thus found widespread use in basic research and recently also entered the clinical arena. Now, in this issue of the JCI, 13 years after introducing HIV-1-based lentiviral vectors (14), the pioneering group led by Luigi Naldini has explored two opposite poles of lentiviral vector design: modifications that can cause cancer via insertional mutagenesis and configurations that greatly increase biosafety when compared with conventional $\gamma$-retroviral vectors (15).

\section{Genetic determinants of lentiviral vector-mediated genotoxicity}

In their study in this issue of the JCI, Montini et al. (15) investigate the contribution of various genetic elements of lentiviral and $\gamma$-retroviral vectors to insertional mutagenesis. The authors used a tumorprone knockout mouse model (animals lacking the tumor suppressor gene cyclindependent kinase inhibitor $2 \mathrm{~A}$ [Cdkn2a]) to demonstrate that lentiviral vectors can act as insertional mutagens provided that the vectors are designed to contain strong enhancer-promoter sequences in their long terminal repeats (LTRs) (Figure 1). Importantly, the authors also demonstrate that the genotoxic risk of lentiviral vectors is significantly lower than that of currently used $\gamma$-retroviral vectors. Furthermore, they show that improvements in vector design, such as self-inactivating (SIN) LTRs, greatly reduce the genotoxic risk in both lentiviral and $\gamma$-retroviral vectors (Figure 1). These data strongly support the adoption of vectors with SIN LTRs for clinical trials of gene therapy. Previous studies pointed in this direction but lacked final evidence in a model with a tumor end point (16-22). Further variables that must be evaluated as part of a risk assessment prior to clinical application of integrating gene vectors include the type and number of target cells involved, the medical history and underlying disease of the patient, and the risk profile of alternative therapeutic approaches.

The study by Montini et al. (15) arrives right in time to have a major impact on the preparation of numerous clinical trials exploring the therapeutic potential of lentiviral vectors in the treatment of inborn or acquired diseases. As is often the case with seminal findings, this study also triggers a number of new questions. Below we discuss the impact of these new data on our understanding of the biology of HIV-1, the role of tumor-prone animal models in the preclinical safety evaluation of lentiviral vectors, and their potential as tools for experimental oncology. 


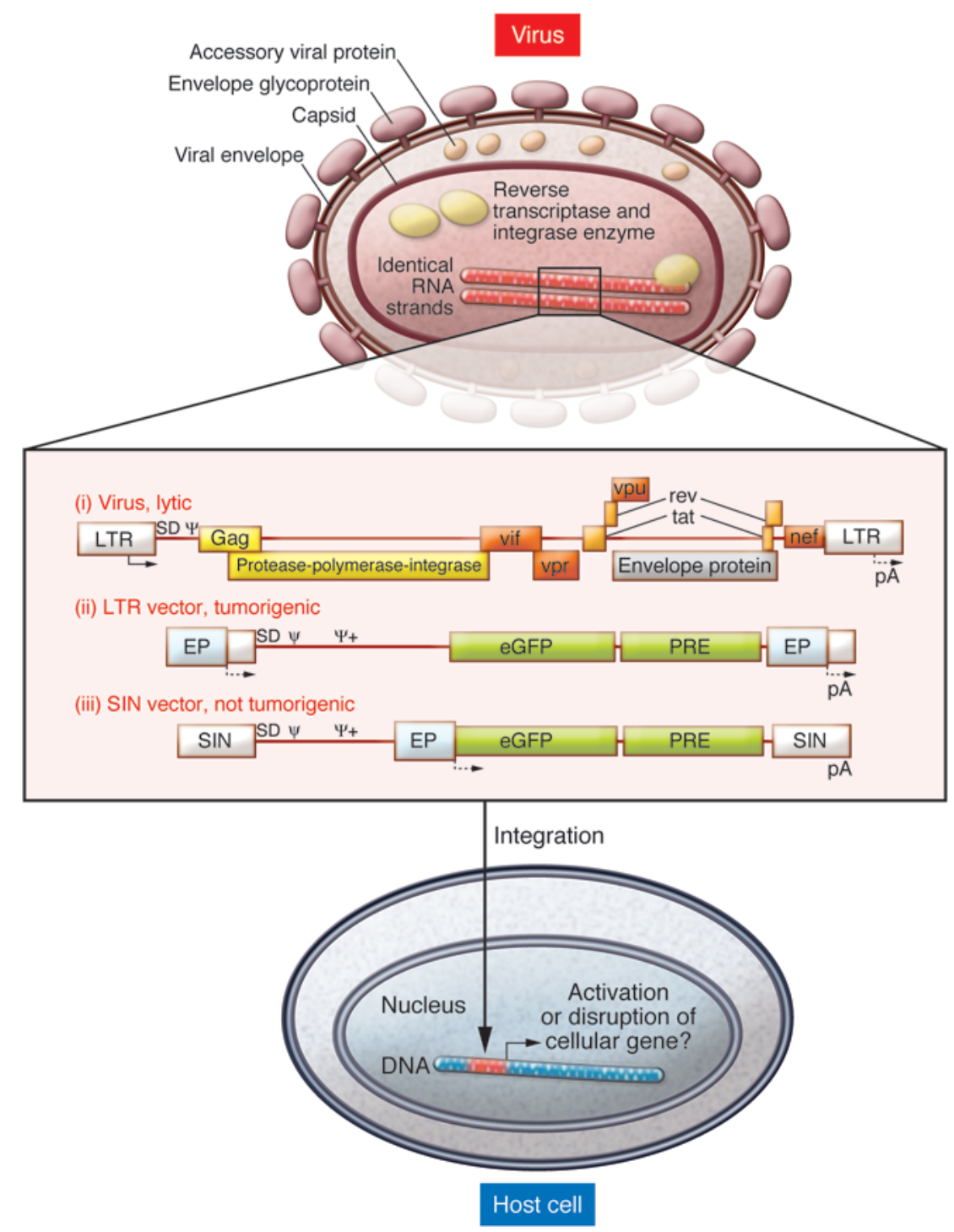

\section{Figure 1}

Oncogenic potential of integrating gene vectors. Schema of a lentiviral particle with its mRNA genome embedded in the capsid is shown. Upon cell infection, viral mRNA is reverse transcribed into double-stranded DNA and transported into the nucleus. Chromosomal integration is mediated by the viral integrase and associated cellular proteins. Integration site selection is semirandom, with a preference for active transcription units. Adjacent cellular genes can be activated or disrupted, depending on interaction with the cis-regulatory sequences of the integrated virally transmitted DNA. Wild-type HIV-1 expresses several structural and regulatory proteins that kill cells by various cytopathic effects (i). The study by Montini et al. (15) in this issue of $\mathrm{JCl}$ shows that lentiviral vectors engineered to express a gene cassette of interest (shown here as enhanced GFP [eGFP] followed by a posttranscriptional regulatory element [PRE]) can be tumorigenic if a strong enhancer-promoter (EP) is introduced into the LTR (ii). Gene activation may involve a splice event through the splice donor (SD) present in the vector backbone and insufficient termination at the polyadenylation signal (pA). Vectors with a SIN LTR carry the EP in an internal position and were not tumorigenic in the Cdkn2a knockout mouse model (iii). Potential oncogenic consequences of gene disruption or internal EP sequences interacting with adjacent cellular promoters remain to be determined. Arrows denote transcriptional start sites; $\Psi$, packaging signal; $\Psi+$, extended $\Psi$; Gag, group-specific antigen; vif, virulence factor; vpr, viral protein R; vpu, viral protein U; rev, regulator of expression of viral proteins; tat, transactivator; nef, negative factor.

\section{Why does HIV-1 not transform cells by insertional mutagenesis?}

In patients infected with HIV-1, uncountable genomic insertion events occur in the major natural target cells, which are mostly $\mathrm{CD}^{+}{ }^{+} \mathrm{T}$ cells and macrophages (23). In theory, almost every location in every gene should be affected even if the viral integration profile has certain preferences and minor "aversions." Montini et al. (15) showed that lentiviral vectors, when equipped with strong enhancerpromoters in their LTRs, may upregulate cellular protooncogenes. In line with another study (22), they demonstrate a mechanism involving a fusion transcript that originates from the lentiviral splice donor, ignores the lentiviral transcriptional termination site, and splices into exons of cellular protooncogenes (Figure 1). Long-distance enhancer effects may also occur. HIV-1 has a very simi- lar insertion pattern (13) and uses the same splice donor and the same weak termination signals as the vectors examined in the present study. This leaves three explanations for the failure of HIV-1 to transform its host cells by insertional mutagenesis. First, the enhancerpromoter sequences of the natural virus may be too weak to induce fusion transcripts into adjacent cellular genes. Indeed, HIV-1 uses a relatively modest enhancer-promoter and enhances gene expression by its mRNA-processing accessory proteins Tat and Rev. Second, the target cells of HIV-1 are inherently more refractory to transformation than the hematopoietic cells targeted by Montini et al. Recent work revealed $\mathrm{T}$ lymphocytes to be more resistant to transformation than more primitive hematopoietic cells $(11,24)$. Third, and probably most relevant, HIV-1 killing of target cells involves a variety of toxic proteins that need to be expressed in the late stages of the viral life cycle (25), thus efficiently eliminating even clones with the potential to activate growth-promoting and antiapoptotic pathways. If the latter is the most relevant explanation, this in turn implies that medical interventions blocking toxic proteins of HIV-1 may bear a residual risk of uncovering insertional side effects. This concern addresses certain principles of "intracellular immunization," in which gene transfer counteracts HIV proteins without eliminating the viral LTRs or blocking viral entry (26).

\section{Tumor-prone mouse models in gene therapy}

The major intention of the work by Montini et al. (15) was to address whether the lentiviral insertion pattern has a lower risk of inducing oncogenic insertional effects 
than the $\gamma$-retroviral insertion pattern. In a previous study conducted in the same model, this conclusion was suggested but not definitively proven, since the vectors that were compared had major differences in the type and position of their enhancerpromoter sequences (27). These same variables were identified by other investigators as crucial determinants of insertional gene dysregulation and cell transformation in vitro (16-20). In their tumor-prone mouse model, Montini et al. now provide evidence that the lentiviral insertion pattern reduces the risk of cell transformation by a factor of 10 , as compared with similarly designed $\gamma$-retroviral vectors. This magnitude became apparent when the risk of tumor induction was corrected for the number of vector integrations in the tumor genomes. The authors thus developed a hazard function that shows a much greater difference in the tumorigenic risk than suggested by the traditional Kaplan-Meier survival curve. Here, we are left with some uncertainties.

Is this factor indicative of a similar increase in biosafety in a clinical trial, i.e., can 10 times more cells or patients be treated before a potential oncogenic event can be detected? Or does this factor rather underestimate the reduction of risk associated with the switch to a lentiviral vector system? More specifically, what will be the additional gain of safety obtained with potentially therapeutic SIN vectors designed to express a gene of interest under control of a more physiologic internal promoter?

Surprisingly, Montini et al. did not observe genotoxic effects from lentiviral SIN vectors containing a strong internal enhancer-promoter (15). This is in contrast with the results of other models that have demonstrated the potential of such a design, at least in the $\gamma$-retroviral context, to transform cells in vitro and in vivo $(16,28)$. Is this the result of the lentiviral insertion pattern or are there factors that may limit the sensitivity of the tumor-prone model?

One interesting finding of the careful analysis of insertional leukemias that occurred in clinical phase 1 trials to treat $\mathrm{X}$-linked SCID by an LTR-driven $\gamma$-retroviral vector was the development of genomic instabilities in the leukemic clones that also contained deletions of the CDKN2A locus, among others $(9,12)$. However, in these leukemias, the loss of tumor suppressor genes is most likely a secondary event, while in the tumor-prone mouse model used here (2), Cdkn2a inactivation represents the "first hit." Thus, in the tumor- prone model, genome instability may occur from the point of formation of the embryo, potentially creating a substantial heterogeneity of cell mutants prior to gene transfer. This may well explain the rapid tumor onset in this model, creating a high background of spontaneous tumors on which the genotoxic impact of relatively weak insertional mutagens can hardly be determined. The very careful insertional genomics studies performed in the current study partially overcome this hurdle (15). Still, to lower the detection limit and reduce the number of animals required to reach statistical significance, further optimizations of genotoxicity assays for gene therapy would be welcome (29).

\section{Lentiviral vectors in basic oncology}

The current study by Montini et al. (15) opens up another important avenue for further investigation (as discussed by the authors): lentiviral vectors specifically designed to trigger insertional mutagenesis may be developed as novel tools to discover cellular protooncogenes whose activation can transform various types of resting cells in vivo, complementing and expanding other approaches, such as those based on cancer-inducing transposons (30). Thus, defining vector configurations with a low risk of insertional protooncogene activation will become the gold standard for somatic gene therapy, whereas developing lentiviral vectors specifically designed to crosstalk with neighboring genes may be established as a novel elegant tool for cancer research.

\section{Acknowledgments}

The authors are grateful for support of their studies by the German Ministry for Research and Education (projects TreatID and iGene), the Deutsche Forschungsgemeinschaft (SPP1230 Ba 1837 and Excellence Cluster REBIRTH), the European Union (projects CONSERT and PERSIST), the National Cancer Institute (5R01CA 107492-02), and the United States Food and Drug Administration.

Address correspondence to: Christopher Baum, Hannover Medical School, Experimental Hematology, Carl-Neuberg-Str. 1, 30625 Hannover, Germany. Phone: 49-511532-6069; Fax: 49-511-532-6068; E-mail: baum.christopher@mh-hannover.de.

1. Wu, X., Li, Y., Crise, B., and Burgess, S.M. 2003. Transcription start regions in the human genome are favored targets for MLV integration. Science. 300:1749-1751.
2. Cattoglio, C., et al. 2007. Hot spots of retroviral integration in human CD34+ hematopoietic cells. Blood. 110:1770-1778.

3. Uren, A.G., Kool, J., Berns, A., and van Lohuizen, M. 2005. Retroviral insertional mutagenesis: past, present and future. Oncogene. 24:7656-7672.

4. Williams, D.A., Lemischka, I.R., Nathan, D.G., and Mulligan, R.C. 1984. Introduction of new genetic material into pluripotent haematopoietic stem cells of the mouse. Nature. 310:476-480.

5. Li, Z., et al. 2002. Murine leukemia induced by retroviral gene marking. Science. 296:497.

6. Hacein-Bey-Abina, S., et al. 2003. LMO2-associated clonal $\mathrm{T}$ cell proliferation in two patients after gene therapy for SCID-X1. Science. 302:415-419.

7. Schwarzwaelder, K., et al. 2007. Gammaretrovirusmediated correction of SCID-X1 is associated with skewed vector integration site distribution in vivo. J. Clin. Invest. 117:2241-2249.

8. Deichmann, A., et al. 2007. Vector integration is nonrandom and clustered and influences the fate of lymphopoiesis in SCID-X1 gene therapy. J. Clin. Invest. 117:2225-2232.

9. Howe, S.J., et al. 2008. Insertional mutagenesis combined with acquired somatic mutations causes leukemogenesis following gene therapy of SCID$\mathrm{X} 1$ patients. J. Clin. Invest. 118:3143-3150.

10. Aiuti, A., et al. 2007. Multilineage hematopoietic reconstitution without clonal selection in ADASCID patients treated with stem cell gene therapy. J. Clin. Invest. 117:2233-2240.

11. Recchia, A., et al. 2006. Retroviral vector integration deregulates gene expression but has no consequence on the biology and function of transplanted T cells. Proc. Natl. Acad. Sci. U. S. A. 103:1457-1462.

12. Hacein-Bey-Abina, S., et al. 2008. Insertional oncogenesis in 4 patients after retrovirus-mediated gene therapy of SCID-X1. J. Clin. Invest. 118:3132-3142.

13. Schroder, A.R., et al. 2002. HIV-1 integration in the human genome favors active genes and local hotspots. Cell. 110:521-529.

14. Naldini, L., et al. 1996. In vivo gene delivery and stable transduction of nondividing cells by a lentiviral vector. Science. 272:263-267.

15. Montini, E., eta al. 2009. The genotoxic potential of retroviral vectors is strongly modulated by vector design and integration site selection in a mouse model of HSC gene therapy. J. Clin. Invest. 119:964-975.

16. Zychlinski, D., et al. 2008. Physiological promoters reduce the genotoxic risk of integrating gene vectors. Mol. Ther. 16:718-725.

17. Evans-Galea, M.V., et al. 2007. Suppression of clonal dominance in cultured human lymphoid cells by addition of the cHS4 insulator to a lentiviral vector. Mol. Ther. 15:801-809.

18. Modlich, U., et al. 2006. Cell-culture assays reveal the importance of retroviral vector design for insertional genotoxicity. Blood. 108:2545-2553.

19. Ryu, B.Y., et al. 2008. An experimental system for the evaluation of retroviral vector design to diminish the risk for proto-oncogene activation. Blood. 111:1866-1875.

20. Hargrove, P.W., et al. 2008. Globin lentiviral vector insertions can perturb the expression of endogenous genes in beta-thalassemic hematopoietic cells. Mol. Ther. 16:525-533.

21. Ramezani, A., Hawley, T.S., and Hawley, R.G. 2008. Combinatorial incorporation of enhancer-blocking components of the chicken beta-globin 5'HS4 and human T-cell receptor alpha/delta BEAD-1 insulators in self-inactivating retroviral vectors reduces their genotoxic potential. Stem Cells. 26:3257-3266.

22. Bokhoven, M., et al. 2009. Insertional gene activation by lentiviral and gammaretroviral vectors. J. Virol. 83:283-294.

23. Goodenow, M.M., and Collman, R.G. 2006. HIV-1 coreceptor preference is distinct from target cell tropism: a dual-parameter nomenclature to define 
viral phenotypes. J. Leukoc. Biol. 80:965-972.

24. Newrzela, S., et al. 2008. Resistance of mature T cells to oncogene transformation. Blood. 112:2278-2286.

25. Costin, J.M. 2007. Cytopathic mechansims of HIV-1. Virology. 4:1-22.

26. Rossi, J.J., June, C.H., and Kohn, D.B. 2007. Genetic therapies against HIV. Nat. Biotechnol. 25:1444-1454.
27. Montini, E., et al. 2006. Hematopoietic stem cell gene transfer in a tumor-prone mouse model uncovers low genotoxicity of lentiviral vector integration. Nat. Biotechnol. 24:687-696.

28. Modlich, U., et al. 2008. Leukemia induction after a single retroviral vector insertion in Evi1 or Prdm16. Leukemia. 22:1519-1528.
29. Nienhuis, A.W., Dunbar, C.E., and Sorrentino, B.P. 2006. Genotoxicity of retroviral integration in hematopoietic cells. Mol. Ther. 13:1031-1049.

30. Dupuy, A.J., Akagi, K., Largaespada, D.A., Copeland, N.G., and Jenkins, N.A. 2005. Mammalian mutagenesis using a highly mobile somatic Sleeping Beauty transposon system. Nature. 436:221-226.

\title{
Wnt therapy for bone loss: golden goose or Trojan horse?
}

\author{
Greg $\mathrm{H}$. Enders
}

Department of Medicine, Epigenetics and Progenitor Cell Program, Fox Chase Cancer Center, Philadelphia, Pennsylvania, USA.

\begin{abstract}
The Wnt pathway has been found to play a role in the development of many tissues and to spur growth and differentiation of adult osteoblasts, sparking interest in its potential clinical application for bone growth. However, when deregulated, this pathway can be oncogenic in some tissues. In this issue of the JCI, Kansara and colleagues reveal that Wnt inhibitory factor 1 is epigenetically silenced in human osteosarcomas and that its absence augments osteosarcoma formation in mice (see the related article beginning on page 837). These observations suggest the need for caution in stimulating the Wnt pathway for therapeutic bone growth.
\end{abstract}

Bone loss is a significant clinical concern. It can be caused by aging or by several diseases and their treatments, such as glucocorticoid hormone therapy for autoimmune disease $(1,2)$. Hip fracture in the elderly, an important complication of bone loss, carries a 1-year mortality rate of approximately $25 \%$ $(3,4)$. Treatment and prophylaxis of bone loss has focused on supporting bone mineralization and inhibiting bone resorption, but attention has increasingly turned to building bone by augmenting osteoblast function $(5,6)$. Some drugs under development act in part through stimulating the Wnt signaling pathway, known to drive osteoblast proliferation and commitment $(6,7)$. In particular, efforts are underway to block the actions of secreted Wnt antagonists such as sclerostin (5). The goal of taking advantage of our burgeoning knowledge of signaling pathways in order to build tissue in general and repair bones in particular has great appeal. The theoretical potential exists for the Wnt pathway to serve as a golden goose, generating new bone indefinitely.

Conflict of interest: The author has declared that no conflict of interest exists.

Nonstandard abbreviations used: APC, adenomatous polyposis coli; SFRP, secreted Frizzled-related protein; WIF, Wnt inhibitory factor.

Citation for this article: J. Clin. Invest. 119:758-760 (2009). doi:10.1172/JCI38973.

\section{The Wnt pathway}

The Wnt pathway is a workhorse of development in multicellular organisms. It directs fate decisions, big and small, such as forming a principal axis of frog embryo development (8) or sculpting heart valves (9). Wnt signaling often drives tissue formation. This functionality endures in some adult tissues that require continuous replenishment, such as the renewal of the intestinal epithelium (10).

However, the Wnt pathway is also the prototypical developmental pathway deregulated in cancer (11). In fact, the pathway's name embodies this potential: Wnt is a contraction of Wingless from Drosophila and Int1 from mammals (12). The Wingless gene was discovered as the site of a mutation in Drosophila responsible for defective patterning of the trunk (13). Int1, the first mammalian homolog of Wingless, was discovered as a common site of integration of mouse mammary tumor virus genomes in tumors induced by the virus (14). These insertion events mediate Wnt1 overexpression and tumor growth. The tendency of Wht deregulation to foster neoplasia is a concern.

The canonical Wnt pathway is liberally endowed with regulatory steps (Figure 1). Wnts, the ligands, are low-abundance secreted factors that are somewhat lipophilic $(15,16)$. For a long time, Wnts could not be isolated biochemically, even when overexpressed in cell culture. Expression was inferred from their biological effects (16). These properties likely reflect the role of Wht proteins as short-range paracrine factors, thought to often be present in steep gradients of abundance. Wnt proteins bind 7-pass transmembrane domain receptors of the Frizzled family (11). Such binding is antagonized by the Wnt inhibitory factors (WIFs) and secreted Frizzled-related proteins (SFRPs), which are all secreted proteins that compete with receptors for ligand binding. Ligand binding activates the Frizzled receptors, transducing a signal through the scaffolding protein Dishevelled and Casein kinase 1 to a protein complex that contains the kinase glycogen synthase kinase- 3 and the tumor suppressor proteins adenomatous polyposis coli (APC; named for the human genetic disease also termed familial adenomatous polyposis) and axis inhibition protein 1 . The APC complex constitutively targets $\beta$-catenin for ubiquitination and degradation. The Wnt signal inhibits the APC complex, leading to stabilization of $\beta$-catenin and its accumulation in the nucleus. There, $\beta$-catenin teams with $\mathrm{T}$ cell factor transcription factors to drive expression of genes such as $c-M y c$ and Cyclin D1 that support cell growth, proliferation, and survival. The pivotal role of Wnt signaling in normal and abnormal growth is underscored by the fact that multiple pathway components have been implicated as either oncogenes or tumor suppressors (Figure 1).

\section{WIF1 and osteosarcoma}

In this issue of the JCI, Kansara et al. (17) examined genes that were epigenetically silenced in osteosarcoma with the notion that these may be important tumor sup- 